\title{
Ecoturismo y turismo cultural: impactos positivos y negativos en el departamento de Boyacá, Colombia
}

\author{
Ecoturism and cultural tourism: positive and negative impacts in \\ the department of Boyacá, Colombia
}

\author{
Nini Johanna Cruz Rodríguez* \\ Universidad Pedagógica y Tecnológica de Colombia (UPTC), Colombia
}

\section{RESUMEN}

Se presentan diversos enfoques sobre el turismo, ecoturismo, turismo cultural y sus impactos positivos y negativos en el departamento de Boyacá, Colombia; región reconocida por su riqueza ambiental y memoria histórica. Mediante el análisis y sistematización de los resultados, de investigaciones desarrolladas durante los últimos tres años, se describen los efectos medioambientales socioculturales y económicos generados por las actividades ecoturísticas. La investigación es cualitativa de nivel descriptivo y método bibliográfico. Se resaltan factores necesarios como punto de partida para el desarrollo de un turismo que permita el disfrute de las generaciones actuales, sin que exista detrimento para las generaciones futuras.

Palabras clave: ecoturismo, turismo cultural, sostenibilidad, Boyacá.

\begin{abstract}
Various approaches are presented on tourism, ecotourism, cultural tourism and their positive and negative impacts in the department of Boyacá, Colombia; a region recognized for its environmental abundance and historical memory. By analyzing and systematizing the results of the research carried out during the last three years, the socio-cultural and economic environmental effects generated by ecotourism activities are described. The research is qualitative, of a descriptive level and bibliographic method. Necessary factors are highlighted as a starting point for the development of tourism activity that allows the enjoyment of current generations, without detriment to future generations.
\end{abstract}

Keywords: ecotourism, cultural tourism, sustainability, Boyaca.

Este es un artículo Open Access publicado bajo la licencia Creative Commons Atribución 4.0 Internacional (CC-BY 4.0).

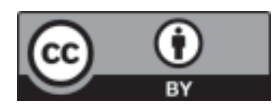




\section{Introducción}

En términos generales, el turismo es reconocido como un mecanismo sustentable para el desarrollo de las regiones, sobre todo, en aquellas con abundantes recursos por explotar, como lo es el caso del departamento de Boyacá en Colombia, reconocido por su potencial cultural y ecoturístico. El ecoturismo, turismo de campo, turismo suave, turismo alternativo y muchos otros términos describen la actividad del turismo en áreas rurales. Y, el denominado turismo cultural, turismo del patrimonio o turismo de intangibles es el redescubrimiento de la riqueza cultural de una región. Por la vocación agrícola y acervo histórico, propio de este departamento, es necesaria la revisión de documentos e investigaciones realizadas en diversos países del orbe durante los últimos tres años; así como, el análisis de los impactos positivos y negativos que tienen sobre el medioambiente y el contexto socioeconómico donde se desarrollan. El objetivo es crear lineamientos que propendan al establecimiento de un turismo que permita el disfrute de las generaciones presentes, sin comprometer la posibilidad que generaciones futuras también puedan hacerlo.

\section{Metodología}

Este estudio tiene un enfoque cualitativo, de nivel descriptivo y método bibliográfico. Toma como punto de partida conclusiones de investigaciones desarrolladas acerca de ecoturismo y turismo cultural. El análisis se realiza desde la corriente teórica del postmodernismo en la cual el turismo es prefigurativamente postmoderno, dada su particular combinación de lo visual, lo estético, lo comercial y lo popular. Para ello, se utilizó la técnica de análisis íntertextual e intratextual, empleando la ficha bibliográfica como instrumento. En el procedimiento se seleccionó cincuenta artículos, publicados en los últimos tres años, de revistas científicas en idiomas español, inglés y portugués. Abordar este tema es pertinente por su evidente aplicabilidad en el departamento de Boyacá, donde se pueden implementar actividades ecoturísticas y culturales en los distintos municipios. Además, se destacan los impactos positivos y negativos en el desarrollo de proyectos ecoturísticos o de turismo cultural. Con información sobre la experiencia internacional en este tema hallada en las bases de datos (Science Direct, Scopus, SciELO, EBSCO, Dialnet y Redalyc) se establecen criterios que propendan al mejoramiento del turismo en los municipios de Boyacá.

\section{Marco referencial}

\section{Boyacá}

El departamento de Boyacá, con su capital Tunja, está ubicado en el centro oriente de Colombia, tiene una superficie de $23189 \mathrm{~km}^{2}$. Debido a su topografía presenta climas diversos, con zonas cálidas y otras frías, incluso con nevados, lo que le permite competir en el sector turístico ya que puede ofrecer atractivos multiculturales y biodiversos; además, cabe resaltar, que es estandarte de la diversidad cultural, histórica y arquitectónica de Colombia (Rodríguez, 2017; Triviño, 2020). Tiene una proyección de 1287032 habitantes, según cifras del Departamento Administrativo Nacional de Estadistica (DANE) para el año 2020, distribuidos en 123 municipios; en donde las tendencias por grupos de edad y sexo muestran notoria disminución de la población, mayoritariamente de las áreas rurales. La migración principalmente de los jóvenes a las cabeceras municipales o a las ciudades del 
departamento es una variable relevante en este análisis. Se relaciona directamente con el imaginario de sus habitantes, quienes señalan que en el campo no hay oportunidades laborales, que no se puede alcanzar mejora en la calidad de vida y que el desarrollo del potencial turístico con el que cuenta la región solo lo pueden lograr personas que vengan de otros sitios (Gobernación de Boyacá, 2018).

Boyacá, además, cuenta con atractivos únicos en el país, reconocidos por su valor histórico, religioso, arquitectónico, paisajístico y ambiental: Tota y Mongua, con dos lagos naturales de majestuosa belleza; Güican y El Cocuy, con sus picos nevados y ecosistemas dignos de ver por todo el mundo; Pisba y Paya, con sus páramos; Tunja, con su historia y sitios religiosos; Villa de Leyva, Monguí o Tópaga, con su arquitectura y riqueza arqueológica; Nobsa, con sus zonas vinícolas; Ventaquemada con el puente de Boyacá o Tipacoque y Soatá, con su cañón del Chicamocha, son unos pocos ejemplos de su riqueza (Bernal, 2016). Además, Boyacá cuenta con aguas termales en municipios como Paipa (Matiz-León, 2019) e Iza (Calderón, 2018), que son estudiados y monitorizados por el Servicio Geológico Colombiano y que atraen una gran cantidad de turistas nacionales e internacionales por las bondades medicinales que se les atribuye (Calderón, 2019; Diaz, 2018); pero, hay que tener en cuenta que de estas fuentes de aguas mineralizadas emanan gases como el radón que, con exposición repetitiva, pueden generar daño en la salud (Díaz Lagos et al., 2019; Moreno, 2018).

\section{Turismo}

El turismo forma parte de uno de los principales sectores económicos en el mundo y opera como fuente de oportunidades para la modernización socioeconómica y cultural de un área geográfica, por tanto, los recursos culturales, naturales y patrimoniales forman una red de gran valor y riqueza, que debe ser planificada, diseñada, gestionada, promocionada y comercializada de forma sostenible con el objetivo de mejorar el desarrollo socioeconómico de las poblaciones locales, y sobre todo, buscando la conservación de los recursos (Marquez y Casas, 2017; Orgaz, 2016; Pagliara et al., 2020). La demanda turística a nivel mundial viene cambiando la motivación de los viajes, más aún con las circunstancias actuales de pandemia que requiere de nuevos paradigmas; primordialmente se buscan desde la oferta nuevas experiencias que satisfagan las necesidades de los turistas por medio de productos distintos al turismo de sol y playa (Cànoves, 2016; Collado, 2017). Aparecen motivaciones relacionadas con la protección y reconocimiento de la naturaleza y todos sus servicios ecosistémicos, lo que da lugar al ecoturismo (Choi, 2020; Çigdem, 2020; Li, 2020); y el conocimiento de la cultura local o turismo cultural (Liu, 2020; Richards, 2018).

\section{Ecoturismo}

La visión de conservación de planeta y sus recursos viene cambiando de acuerdo a la necesidad imperiosa de cuidarlo y protegerlo de la propia humanidad; del mismo modo, el concepto de turismo se viene modificando y específicamente el concepto de ecoturismo. Según refiere Zapata (2009, como se citó en Córdova, 2020) «es toda forma de turismo basado en la naturaleza, por la que la motivación principal de los turistas es la observación y apreciación de la naturaleza y las culturas tradicionales, dominantes en las zonas naturales». En el Programa de las Naciones Unidas para el Medio Ambiente (2017, como se citó en Córdova, 2020) es considerado como el «segmento del mercado de turismo en el que los viajeros tienen como interés específico 
contemplar y conocer las interacciones entre la biodiversidad, los paisajes, las zonas históricas, así como las diversas manifestaciones de las poblaciones locales». Mientras la Sociedad Internacional de ecoturismo (2020, como se citó en Córdova, 2020) enfatiza que ecoturismo es «viajar en forma responsable hacia áreas naturales, conservando el ambiente y mejorando el bienestar de las comunidades locales medio ambiente por parte de los visitantes y de los pobladores locales».

Asimismo, Strasdas (2000, como se citó en Padilla, 2019) lo define como «todas las formas de turismo que tienen lugar en áreas naturales y para las que la vida natural, en alguna de sus formas, ejerce atracción especial» y que «implica la conservación y el respeto de los recursos y valores naturales, cuya existencia ha de ser garantizada para el sostenimiento de la actividad y asegurar el disfrute del medio por parte de las generaciones futuras» (Vanegas, 2006, como se citó en Padilla, 2019). Es «una alternativa de desarrollo a través de la participación y empoderamiento de la ciudadanía sobre el control de sus recursos y la oferta de productos, garantizando la conservación de los ecosistemas y el dinamismo de la economía local» (De la Torre, 2018, como se citó en Padilla, 2019).

Por lo expuesto, es necesaria la protección de la naturaleza en el afán productivo de su usufructo y, al tratarse de recursos que no son autorrenovables es vital el diseño, planeación y ejecución de estructuras empresariales que propendan el disfrute de las generaciones presentes, sin limitar la posibilidad que las futuras generaciones también lo puedan hacer.

\section{Turismo cultural}

El concepto de turismo cultural se ha modificado a lo largo del tiempo, se «remite al conjunto de actuaciones programadas con el objetivo de conseguir una óptima conservación de los bienes patrimoniales y un uso de estos bienes adecuado a las exigencias sociales contemporáneas» (Ballart, 2001, como se citó en Díaz, 2019). Es así que el turismo cultural se desarrolla como «la articulación de los procesos de identificación, valoración, competitividad y difusión del patrimonio cultural, con el fin de generar beneficios económicos garantizando la sostenibilidad de los sectores cultura y turismo» (Ministerio de Cultura y Turismo de Colombia, 2007, como se citó en Díaz, 2019).

Toselli (2019, como se citó en Díaz, 2019) se refiere al espacio de producción del turismo cultural:

[Es] uno de los ámbitos más novedosos en las iniciativas locales, ya que supone una visión sobre los recursos culturales e históricos como activos de desarrollo, y por esto permite incorporar elementos de diferenciación tanto en los procesos productivos como en los productos locales.

Dentro de esta modalidad de turismo convergen distintas motivaciones de búsqueda cultural, como el turismo gastronómico (Pavlidis y Markantonatou, 2020; Seyitoglu e Ivanov, 2020); turismo religioso (Luz, 2020; Terzidou, 2020); turismo de museo (Carbone et al., 2020) y turismo patrimonial propiamente dicho, en el que se considera la cosmovisión cultural; es decir, los turistas aprenden, descubren, perciben e interpretan información cultural relacionada a cada sitio visitado, disfrutando de la experiencia cultural con bienes tangibles e intangibles como danzas, cerámica, cestería y otros más (Wei et al., 2020). 
El turismo es sostenible cuando es capaz de generar un desarrollo compatible tanto con las necesidades del destino turístico como con sus limitaciones económicas, y por ello, debe existir un equilibrio entre las necesidades de los turistas y las comunidades locales con los ecosistemas. Se generan efectos positivos y negativos en el medioambiente y en el contexto socioeconómico del área donde están presentes (Weaver et al., 2020).

Por lo tanto, el turismo cultural se presenta como una estrategia económica que puede ser implementada en cualquier región del mundo. Para lograrlo, se requiere la acción conjunta y coordinada de los organismos de gobierno para que establezcan leyes que ordenen la protección de los sitios históricos, protegiendo las riquezas -de todo tipo- que se encuentran en cada lugar. Además, es fundamental la participación de grupos empresariales que valoran la importancia de las comunidades y sus patrimonios históricos representados en bailes, comidas, artes manuales y muchas otras manifestaciones.

\section{Impactos positivos generados por actividades ecoturísticas}

Es evidente el impacto significativo ambiental, sociocultural y económico generado por las actividades ecoturísticas. Básicamente, se destaca el fortalecimiento de la capacidad de asociación de las comunidades para ofrecer los servicios; mayor interacción con el otro; reafirmación de valores y de la identidad cultural (Minnaert, 2020); así como, la resignificación de manifestaciones culturales y el respeto por estas. Las operaciones turísticas deben promover el comportamiento respetuoso hacia las culturas y poblaciones locales (Bires y Raj, 2020).

Se consideran impactos positivos el bienestar de los hogares residentes por el acceso a servicios, instalaciones, infraestructura, educación y equipamiento turístico (Wang et al., 2020). Estos, y otros más, que favorecen a los habitantes del departamento de Boyacá, pues como se describe más adelante, no se trata solamente de obtener más dinero sino de rescatar y proteger el patrimonio de las regiones.

En un estudio de caso de la población de Qinggangshu, en China, se evidencia que la reestructuración espacial rural impulsada por el ecoturismo es el resultado de fuerzas internas y externas, lo que puede acelerar la consolidación de los territorios y conducir a la revitalización rural; por lo contrario, en aquellos municipios sin ninguna dotación de recursos, la simple consolidación de proyectos turísticos no puede mejorar la economía rural en un corto período; sin embargo, depende de la reestructuración espacial rural, la economía rural y la cultura social para su reestructuración (Gao y Cheng, 2020). La participación de los gobiernos municipales boyacenses y sus representantes nacionales son determinantes en el impulso y mantenimiento de programas de ecoturismo, pues sin acompañamiento constante -sobre todo en lo que tiene que ver con gestión de recursoses difícil su consolidación.

Las funciones ecosistémicas de los municipios que acogen el ecoturismo han variado gradualmente, y han pasado de residencias individuales y producción agrícola, a tener lugares que ofrecen alojamiento, compras, restauración, entretenimiento y otras funciones para satisfacer las necesidades de los turistas, y de esta manera los habitantes rurales obtienen mayores oportunidades de empleo (Gao y Cheng, 2020). Así, la contribución del ecoturismo se describe principalmente en términos de ingresos crecientes y creación de empleo, adopción 
de nuevas prácticas de trabajo, mejora de las habilidades empresariales, conservación de la naturaleza, fortalecimiento de la identidad y cohesión de la comunidad. Además, el turismo rural está conectado con muchos servicios, lo que permite la expansión de negocios complementarios como alimentos locales o artesanías locales. Igualmente importante, el turismo rural se ha desarrollado tradicionalmente utilizando propiedades existentes (por ejemplo, casas de campo y propiedades de tierra) como alojamientos turísticos, haciendo posible el mantenimiento de lazos con la propiedad y la tierra por un grupo de personas que de otra manera no vivirían en áreas rurales (Dinis et al., 2019). Por lo que, el desarrollo de proyectos ecoturísticos en el departamento de Boyacá, puede ser una estrategia para lograr disminuir la creciente migración de la población, sobre todo de la más joven, hacia los centros urbanos; además, se debe fortalecer la educación con formación en turismo, destacando el potencial de cada uno de sus municipios.

Precisamente, en la investigación de Guaita et al. (2019), efectuada en España, específicamente en la región mediterránea, se concluye que el desarrollo de la actividad ecoturística puede generar efectos positivos como la creación de empleo, la mejora de la calidad de vida de los lugareños, la mejora de la imagen pública de la región, la preservación del patrimonio cultural e incluso el desarrollo de la red comercial. Adicionalmente, aumento de las oportunidades para que los habitantes disfruten del tiempo libre, mejor valoración del entorno natural, mejoras relacionadas con el transporte público y las instalaciones, interacción cultural, entre otros. Beneficios que recaen en los municipios que hacen parte del denominado valle de Tenza (Tenza, Guateque, Almeida, Garagoa), donde la presencia de aguas termales trajo consigo un aumento significativo de turismo. Situación que obligó a los gobiernos departamental y municipal a mejorar la infraestructura vial y hotelera de la región, logrando un aumento significativo en el número promedio de visitantes por año.

Otro impacto positivo se precisa en la investigación de Pilving et al. (2019), realizada en Estonia, en áreas rurales con poblaciones de menos de 4000 habitantes. Aquí, el ecoturismo a menudo se convierte en un estilo de vida empresarial en el que los empresarios rurales no se centran únicamente en los ingresos, sino en el cumplimiento de sus preferencias de estilo de vida. En este entorno se crean asociaciones que, a pesar de ser por lo general informales, dan prioridad e importancia a las relaciones interpersonales, lo que motiva a las partes interesadas para unirse en una asociación ecoturística.

De igual forma sucede en el departamento de Boyacá, en municipios como Villa de Leyva, Sutamarchan, Nobsa y Tinjaca, en los que el establecimiento de viñedos u olivares como asociación de productores continúa, en primera instancia, con el estilo de vida europeo de algunos de sus fundadores y, en segunda instancia, con la generación de ingresos mediante visitas guiadas por las fincas y cultivos de personas nacidas allí. No solamente se crean viñedos, también se produce quesos madurados, yogures, a cargo de pequeños empresarios de clusters comerciales de los municipios de Paipa, Duitama, Tuta, Sotaquirá. Es decir, se evidencia que es posible crear vida empresarial, tal como lo reflejan los trabajos rurales de Estonia, previamente descritos.

Además, el ecoturismo ofrece beneficios sociales muy importantes como el empoderamiento de las clases sociales vulnerables, la unión de los miembros de la comunidad y las familias, brinda a los residentes un sentido de lugar y sentimiento de hogar; también, se llevan a cabo reuniones para el entretenimiento social, haciendo que la población rural se sienta útil y necesaria. 
Con la práctica del ecoturismo se mejora la infraestructura local, se aviva una mayor conciencia ambiental, y al atraer a los jóvenes de regreso a los campos, se ayuda a satisfacer y mejorar las relaciones familiares y se fortalecen lazos sociales en la sociedad rural, como se demostró en la investigación de Su et al. (2019) en China, región noroccidental. Actividades tradicionales, como la agricultura (incluidas las plantaciones de té) son percibidas tanto por los residentes como por el gobierno local, como una base importante para el desarrollo del ecoturismo; unido al desarrollo turístico, se incrementan las oportunidades de empleo local, extendiendo los beneficios a los participantes no turísticos. Las relaciones sinérgicas entre el turismo, la agricultura y el empleo local reducen la necesidad de migración para buscar trabajo y contribuyen a la retención de la mano de obra rural en los campos.

Para el departamento de Boyacá estos beneficios sociales del ecoturismo son fundamentales para la regeneración y el desarrollo rural sostenible; además, hacen posible crear espacios atractivos para los habitantes urbanos donde la red social puede reconstruirse integrando a las familias en torno a un objetivo común.

\section{Impactos negativos generados por actividades ecoturísticas}

El ecoturismo también puede generar impactos negativos en las áreas rurales, que como ya se mencionó, ocupan la mayor parte del departamento de Boyacá.

Los impactos negativos se manifiestan de diversas maneras, por ejemplo, Gao y Cheng (2020) en un estudio desarrollado en el centro de China observaron que la cultura traída por los turistas urbanos impactó en la cultura rural tradicional. Los conflictos entre pobladores aumentaron al competir por más clientes, el concepto de vida y sistema de valores arraigado en la sociedad local se olvidaron progresivamente; los objetos de identidad cultural fueron desapareciendo y los intercambios culturales intergeneracionales en las zonas rurales se reemplazaron por un fetichismo centrado en el dinero. Una situación similar se presenta en los municipios Villa de Leyva y Ráquira (departamento de Boyacá), el auge del turismo, de distintos tipos, trajo como consecuencia que los turistas con alto poder adquisitivo decidieran tomar residencia allí, generando una urbanización de lo rural en menoscabo de la riqueza visual de los territorios, pues es más frecuente ver casas campestres de estilo moderno y cada vez menos de estilo campesino.

Asimismo, en municipios boyacenses como Tota, Cuitiva y Moniquirá se observan efectos negativos como los ocurridos en la provincia de Toscana, Italia y otras regiones rurales de todo el mundo donde el ecoturismo crece y se convierte en impulsor estable para el desarrollo social y económico. También puede desencadenarse un turismo de masas, provocando un doble efecto dañino: (1) dado que el turismo de masas requiere una expansión acumulada para dar cabida a un mayor número de turistas, en las zonas rurales, sin duda, se comprometerá la belleza del paisaje, recurso local fundamental; (2) el crecimiento acumulado requerido para satisfacer las expectativas de los turistas se caracteriza por la construcción en el campo de centros turísticos, aldeas artificiales y condominios residenciales de alta densidad, con la consiguiente pérdida de identidad cultural tradicional, que también es un elemento clave como atractivo para los paisajes rurales. Por ejemplo, en detrimento de una belleza natural, como lo es el lago de Tota, se ha permitido la práctica de pesca comercial y construcción de lujosos hoteles y centros de alojamiento en sus áreas perimetrales (Randelli y Martellozzo, 2019). 
Por consiguiente, el ecoturismo podría beneficiar predominantemente a personas externas provenientes de orígenen urbano o extranjero, y tener efecto adverso sobre el bienestar de las comunidades locales, particularmente cuando los trabajos disponibles para la población local son servicios de paga menor (Coromoto, 2019). Sumado a ello, el turismo es una fuente de ingresos vulnerable e inestable, con bajas tasas de retorno de la inversión, muy sensible a los cambios reales o percibidos de los destinos y las preferencias de los consumidores (Dinis et al., 2019). Es lo que ocurre en los municipios de Muzo, Otanche y Pauna, que forman parte de la zona occidental del departamento de Boyacá que, cuando se vive un ambiente de paz, el turismo se dinamiza, pero cuando se presentan los conflictos sociales propios de la extracción de esmeraldas, turistas nacionales e internacionales optan por otros destinos; como consecuencia, se perjudica la infraestructura, los resultados son negativos en la balanza de entradas y salidas, ocasionando despido de personal y hasta cierre de algunos centros turísticos.

Además de lo mencionado, Guaita et al. (2019) incluyen como impactos negativos el hacinamiento público de espacios e instalaciones, interrupción del estilo de vida de los lugareños, aumento en los precios de las propiedades, problemas de seguridad, daños ambientales, aumento en la producción de residuos y el uso intensivo de los recursos naturales. Considerando que la intensidad y el origen de los impactos negativos dependen de diferentes factores que condicionan la sostenibilidad del turismo, no solo desde una perspectiva ambiental, sino también desde una perspectiva social y económica. Depende básicamente del número total de llegadas de turistas y el grado de concentración de turistas en ciertas épocas del año. Fenómeno que se presenta en casi todos los municipios de departamento, pues muchos de los ecoturistas solamente tienen la posibilidad de hacerlo en las dos temporadas en las que el trabajo se lo permite (vacaciones de mitad y fin de año). Razones por las que subsistirán aquellas empresas que resistan las épocas de bajo turismo con los ingresos generados en las épocas de alto tráfico de turismo.

Con respecto a la vulnerabilidad de las empresas ecoturísticas Pilving et al. (2019) sostienen que el impacto negativo o factor de alto riesgo aumenta cuando: (1) la idea no está basada en individuos líderes; (2) los objetivos son confusos y los resultados no se miden; (3) la comunicación disminuye; (4) las partes interesadas carecen de tiempo para participar en actividades voluntarias; (5) la financiación es incierta; (6) los cambios institucionales aumentan la incertidumbre en áreas rurales y (7) no existe conexiones con los centros urbanos. En el caso de Bocayá la implementación del programa denominado Anillos Turísticos, en el año 2009, permitió formar una red de asociaciones turísticas regionales centrada en los intereses de la comunidad, en municipios como Chivatá, Toca y Sáchica, pero desafortunadamente nunca se creó la infraestructura turística necesaria y el gobierno local incumplió la mayoría de los ítems anteriormente mencionados. Se generaron expectativas en los habitantes rurales que no recibieron la atención debida, desmotivando a muchos que desestimaron la formación de otras empresas ecoturísticas (Castellanos y Mendoza, 2018).

\section{Impactos positivos generados por el turismo cultural}

El desarrollo del turismo cultural en la última década favorece la conservación de una variedad de bienes culturales tangibles e intangibles, también ayuda al desarrollo de actividades relacionadas con las artes, la artesanía y la creatividad y, aunque anteriormente se le consideraba como una forma de turismo dirigida a las personas con niveles más altos de educación, ahora es un tema muy popular (Liu, 2020). 
En concordancia a ello, el turismo cultural es entendido como un proceso de intercambio social mediante el cual una población puede participar en el consumo de su patrimonio cultural preservado en museos, bibliotecas y archivos, mantenido en jardines botánicos, parques y reservas nacionales expresado en forma visual, artes literarias, escénicas, folklore o las artesanías (Haigh, 2020). De esta manera, se clarifica que el turismo cultural en Boyacá tiene el potencial de expresarse de muchas maneras, aún dentro de un mismo municipio, por lo que para cualquier tipo de demanda existe la oferta suficiente para atenderla y generar una cadena de beneficios para sus habitantes.

Los eventos artísticos también son atracciones turísticas y se les puede considerar como actividades de beneficio mutuo. Los eventos amplían la gama de oportunidades de consumo y entretenimiento disponibles para los turistas, mientras que las atracciones se convierten en una industria turístico-cultural que aumenta la viabilidad financiera de las actividades y promociones culturales municipales al aumentar el patrocinio y, por lo tanto, los ingresos por eventos. Así lo estableció Haigh (2020), mediante un análisis teórico acerca del turismo cultural en Sarawak, estado de Malasia en la isla de Borneo que se extiende por la costa noroeste de la isla e incluye varias playas en el mar de la China Meridional.

Al respecto, coinciden investigadores de China, Canadá y Estados Unidos cuando sostienen que la experiencia del turismo cultural se considera predominantemente, no como estética o intelectual, sino como social. Es que la mayoría de las personas visitan museos y sitios turísticos en grupos y aquellos que los visitan solos regularmente se reúnen y hablan con otros visitantes o miembros del personal. Gran parte de la interacción social en estos contextos es una forma de conectarse entre sí y encontrar significado juntos. La cuestión de las interacciones sociales en las experiencias de turismo cultural es importante al ser un factor primario en los motivos, satisfacción y compromiso de los visitantes (Li et al., 2020; Minnaert, 2020; Pyke et al., 2019).

Otro aspecto positivo del turismo cultural es que los turistas se involucran. Hay una relación directa en la búsqueda de comprender profundamente la cultura local del destino y mayor participación de los visitantes, creándose mejores niveles de contacto e intercambio cultural (Chen y Rahman, 2018).

Además, Chávez (2020) propone cuatro tipos de motivación aplicables al turismo cultural que tienen diferentes respuestas o consecuencias, como diferentes niveles de contacto cultural y participación de los visitantes. Por ejemplo, los turistas con la motivación de regulación intrínseca pueden estar más dispuestos a aprender a fondo sobre una nueva cultura cuando viajan, mientras que los turistas con una motivación extrínseca simplemente viajan por que les gusta hacerlo; probablemente los primeros requieran de mayor contacto cultural. Luego está la motivación de logro, los turistas viajan principalmente para aprender sobre estrategias comerciales eficaces, triunfadoras para aprenderlas y replicarlas; a diferencia de la motivación incentiva, no es el dinero o la ganancia monetaria lo que persiguen, sino crear una empresa de turismo reconocida y autosustentable. En resumen, se presentan diferentes niveles de motivación y respuestas para un destino turístico cultural (Chen y Rahman, 2018).

\section{Impactos negativos generados por el turismo cultural}

Los impactos negativos que se producen por el turismo cultural son estudiados en diferentes ámbitos del mundo. Trabajos de investigación desarrollados en la zona centro de Malasia y suroriental de Taiwán tienen 
como objetivo la promoción de un turismo cultural que se basa en dar a conocer representaciones artísticas de comunidades indígenas. Haigh (2020) y Hunter (2020) plantean que las estrategias de desarrollo basadas en el turismo cultural requieren de un análisis profundo entre las áreas urbanas y rurales para dirigir los recursos eficientemente, de tal manera que los responsables políticos, operadores turísticos y productores culturales (como artistas, escritores e intérpretes) promuevan y permitan el logro de retornos económicos directamente a las comunidades locales. Por otra parte, Fan et al. (2020) establecen que las tribus indígenas terminan finalmente negociando su indigeneidad, conformada por imaginarios globales en poder de los turistas, y por su búsqueda del desarrollo y la modernidad.

Se considera también como impacto negativo del turismo cultural el proceso de desculturalización del destino, que se da en ferias y fiestas municipales acomodadas a celebraciones españolas con corridas de toros o eventos locales escenificados para los turistas, como vendimias o tomatinas; terminan siendo una «autenticidad escenificada» (Díaz, 2019). En consecuencia, se genera una impresión sensible de rechazo, por parte de los habitantes locales, a los sitios de reconocimiento sagrado o a las tradiciones del lugar, pues surge el sentido de protección de sus recursos frente a una utilización que no les arroja dividendos.

Otro impacto es el denominado «choque cultural», el turista puede tener una percepción sesgada de la autenticidad al no tener conocimiento previo de lo que se le muestra, ya que no puede comparar y pensar críticamente sobre los artefactos culturales, perdiendo el principio de transmisión cultural que se pretende. Por ello, es conveniente, previamente a su llegada a los sitios de visita, ponerlos en contexto para que se formen una imagen de lo que experimentarán (Tiberghien et al., 2020). Como recomendación, además, se sugiere el montaje de quioscos interactivos en los lugares de visita para orientar a los visitantes y brindarles las narrativas culturales que incluyen ubicación, riqueza cultural y mucho más de la región en que se encuentran (Wu et al., 2020).

En ese contexto y frente a la creciente necesidad de incorporar componentes virtuales y físicos a la experiencia del turismo cultural, se promueve desde la economía del conocimiento postindustrial la creación de industrias culturales y creativas (ICC), como insumo para que los municipios resalten los sitios patrimoniales, potencien su identidad cultural y fomenten la diversidad cultural (Landoni et al., 2020; Rodríguez-Gulías et al., 2020). Pero, no todos los lugares están preparados para tal fin, existen barreras de tipo tecnológico, mental, social, y paradójicamente, hasta culturales que impiden el desarrollo de la industria cultural al considerar que industria y patrimonio son incompatibles (Sam Liu y Huang, 2020). Por cierto, los niveles de interés de los turistas varían según la complejidad de la infraestructura computacional que se les presenten; la mayoría de los turistas prefieren presentaciones interactivas para contenido relacionado con las estatuas de dioses, objetos, estilo y diseño de los templos, por ejemplo; y prefieren presentaciones animadas con contenido 3-D para lo relacionado a la historia municipal o biografía de personajes históricos (Wu et al., 2020).

Para complementar, debe tenerse en cuenta la estacionalidad en el turismo, ya que generalmente, se generan inconvenientes por el uso poco eficaz de las instalaciones disponibles durante todo el año, la variabilidad de los precios de los productos y servicios, la creación de empleo temporal en el mercado laboral y varios efectos sociales y ambientales. De acuerdo a Vergori y Arima (2020) es ampliamente conocido que la estacionalidad 
está estrictamente relacionada con los atributos del destino turístico (como condiciones climáticas, eventos, atracciones físicas, etc.) y también depende de factores relacionados con los propios turistas y su área de origen (como inercia, vacaciones institucionalizadas y cambio en los gustos).

Por último, son también impactos negativos las amenaza a las relaciones sociales tradicionales, así como a las prácticas y autenticidad cultural para responder a la demanda turística. Como lo atribuyen Cunha et al., (2020) las poblaciones rurales tienen que adaptarse a desafíos adicionales, como la presencia continua de extraños y el desempeño de un nuevo rol productivo, así como a los constantes conflictos en el grupo, pérdida de confianza y reciprocidad hacia los recién llegados como resultado de la migración turística y de servicios.

\section{Conclusiones}

Los impactos positivos del ecoturismo y del turismo cultural se visualizan en la conservación de los ecosistemas estratégicos, el uso sostenible de los espacios naturales y la valoración de las especies en vía de extinción al evitar su comercio ilegal, la diversificación económica, el desarrollo endógeno de las comunidades por la creación de empresa y generación de empleo con fuente constante de ingresos, la adopción de nuevas prácticas de trabajo, la mejora de las habilidades empresariales, la conservación de la naturaleza, el fortalecimiento de la identidad y cohesión de la comunidad, la conservación de bienes culturales tangibles e intangibles y el empuje a las actividades relacionadas con las artes, la artesanía y la creatividad municipal.

Por lo contrario, el desarrollo de prácticas ecoturísticas o de turismo cultural no planificadas puede generar impactos negativos como el resquebrajamiento de la unidad familiar, riesgo social de poblaciones vulnerables, utilitarismo de minorías étnicas, pérdida de propiedad intelectual a partir del conocimiento ancestral, pérdida de identidad y de valores, rechazo al turista, etc. Del mismo modo, la construcción de infraestructura y otros servicios tiene un impacto adverso y directo sobre el medioambiente; se crea una amenaza a los recursos naturales y a la biodiversidad por la caminata repetitiva por los mismos lugares, contaminación visual por generación de residuos sólidos, contaminación auditiva por el sonido de motores, música en vehículos restaurantes y tránsito continuo de personas. También, se observan cambios en el comportamiento de la fauna por ruido, luz excesiva o por acercarse a los humanos para obtener alimento; la contaminación de las corrientes hídricas e incremento en la producción de aguas residuales y emisiones de dióxido de carbono $\left(\mathrm{CO}_{2}\right)$, tiende a aumentar el costo de la tierra y el costo de vida lo cual genera empobrecimiento de la población local, pues mucho del dinero que se genera por el turismo no siempre se queda en los bolsillos de la población residente y los salarios por sus servicios son relativamente bajos. Existe alta estacionalidad lo que no favorece la contratación de los habitantes locales, hay riesgo de insuficiencia alimentaria local ya que se genera un cambio en las actividades productivas, se fomenta pérdida laboral, pues la necesidad de dominar otro idioma en comunidades que no tienen acceso a educación diversa con manejo de idiomas, lleva a buscar personal de otros lugares.

En este sentido, el planeamiento regional es crucial en la dinamización del ecoturismo y el turismo cultural, particularmente en zonas del departamento de Boyacá en las que existe gran potencial de desarrollo pero que por falta de apoyo gubernamental no han logrado consolidarse. 
Asimismo, es fundamental el desarrollo de ecosistemas empresariales sostenibles, en los que no solo se fortalezca el crecimiento de un negocio específico, como los alojamientos de turismo rural, sino que también se potencie el desarrollo de otros negocios relacionados como los de souvenirs, restaurantes, atracciones culturales, etc., para de esta manera formar redes de infraestructura turística.

Con los recursos y herramientas que brindan las TIC (tecnología de la información y la comunicación) los procesos productivos se facilitan. Considerando que los turistas ecoturísticos o culturales son atraídos por actividades interactivas, participativas, con entornos prácticos de aprendizaje, progresivamente las visitas guiadas y los folletos informativos deben ser sustituidos por ideas novedosas y atractivas.

Finalmente, al evaluar estos impactos positivos y negativos del ecoturismo y turismo cultural en el departamento de Boyacá se puede consolidar un turismo sostenible que propenda a que el patrimonio natural y cultural de los municipios se conserve y proteja, garantizando así su permanencia en el tiempo y el disfrute para generaciones actuales y futuras.

\section{Conflicto de intereses}

La autora no tiene conflicto de intereses con el contenido de este trabajo.

\section{Referencias}

Bernal, E. (2016). Turismo de naturaleza y cultura en el campo [Tesis doctoral, Universidad de Salamanca]. https://mail.google.com/ mail/u/0/\#search/dalysferrin\%40gmail.com/15f3309881fe0d8d

Bires, Z. y Raj, S. (2020). Tourism as a pathway to livelihood diversification: Evidence from biosphere reserves, Ethiopia. Tourism Management, 81, 14. https://doi.org/10.1016/j.tourman.2020.104159

Calderón, R. (2019). Determination of Hydrothermal Prospects in the Geothermal Region of Paipa (Boyacá, Colombia ), Using Remote Sensing and Field Data. Earth Sciences Research Journal, 23(4), 265-282.

Calderón, R. A. (2018). Infrarrojo termal en la prospección de fuentes hidrotermales en la región de Paipa-Iza, Boyacá [Tesis de maestría, Universidad Nacional de Colombia]. C:/Users/user/Downloads/1057580892.2019.pdf

Cànoves, G. (2016). Tourism in Spain, beyond the sun and the beach. Recent evolution and changes in coastal destinations towards cultural tourism. Boletin de La Asociacion de Geografos Españoles, 71, 431-454. https://doi.org/10.21138/bage.2289

Carbone, F., Oosterbeek, L., Costa, C. y Ferreira, A. M. (2020). Extending and adapting the concept of quality management for museums and cultural heritage attractions: A comparative study of southern European cultural heritage managers' perceptions. Tourism Management Perspectives, 35, 9. https://doi.org/10.1016/j.tmp.2020.100698

Castellanos, L. y Mendoza, M. (2018). Plan de mejoramiento para la ruta: Anillo turístico de los dinosaurios en el departamento de Boyacá [Tesis pregrado, Universidad Externado de Colombia]. https://bdigital.uexternado.edu.co/handle/001/1384 
Çigdem, K. A., Tülay, C. T., Ferah, Ö. y Hasan, T. (2020). Land use suitability analysis of rural tourism activities: Yenice, Turkey. Tourism Management, 76, 103949. https://doi.org/10.1016/j.tourman.2019.07.003. https://www.sciencedirect.com/science/article/ pii/S0261517719301396

Coromoto, M. (2019). Turismo - Naturaleza - Sostenibilidad: Jornadas de discusión y propuestas. Un aporte desde el CIDE en la Cámara de Turismo del estado Mérida. Visión Gerencial, 2, 333-343. https://www.redalyc.org/articulo.oa?id=465566915015

Collado, M. (2017). Planificación territorial y turística en destinos de sol y playa maduros [Tesis doctoral, Universidad Politécnica de Madrid]. https://doi.org/10.20868/UPM.thesis.48034

Córdova, K. (2020). El turismo rural comunitario y el ecoturismo como alternativas para el desarrollo local de Tupe [Tesis de licenciatura, Universidad Peruana de Ciencias Aplicadas]. Repositorio académico UPC. http://hdl.handle.net/10757/621923

Cunha, C., Kastenholz, E. y Carneiro, M. J. (2020). Entrepreneurs in rural tourism: Do lifestyle motivations contribute to management practices that enhance sustainable entrepreneurial ecosystems? Journal of Hospitality and Tourism Management, 44, 215-226. https://doi.org/10.1016/j.jhtm.2020.06.007

Chávez, L. (2020). Precedentes de motivación, uso y gratificación, como determinantes participativos en entornos turísticos 2.0 y su efecto en el comportamiento interactivo [Tesis doctoral, Universitat de València]. España. https://roderic.uv.es/handle/10550/72558

Chen, H. y Rahman, I. (2018). Cultural tourism: An analysis of engagement, cultural contact, memorable tourism experience and destination loyalty. Tourism Management Perspectives, 26, 153-163. https://doi.org/10.1016/j.tmp.2017.10.006

Choi, M. (2020). Multiple environmental subjects: Governmentalities of ecotourism development in Jeungdo, South Korea. Geoforum, 110, 77-86. https://doi.org/10.1016/j.geoforum.2020.01.011

Departamento Administrativo Nacional de Estadistica (DANE). (2020). Proyecciones de población calculadas con base en los resultados del Censo Nacional de Población y Vivienda - CNPV-2018. https://www.dane.gov.co/index.php/estadisticas-por-tema/demografiay-poblacion/proyecciones-de-poblacion

Díaz, D. (2019). Modelo de gestión para el turismo cultural sostenible en la ciudad de Manizales. International journal of scientific management and tourism, 4(2), 237-248. https://dialnet.unirioja.es/servlet/articulo?codigo=6640380

Díaz, Y. y Morales, J. E. (2018). Plan estratégico en turismo de salud para Instituto de turismo de Paipa - ITP, periodo $2019-2021$ [Tesis de maestría, Universidad Nacional Abierta y a Distancia - UNAD]. https://repository.unad.edu.co/handle/10596/21442

Díaz Lagos, M., Sajo-Bohus, L., Sandoval Garzón, M. A., Vergara Gómez, I. y Martínez-Ovalle, S. A. (2019). Radon concentration in hydrogeothermal deposit and spas of Boyacá, Colombia. Applied Radiation and Isotopes, 145, 131-136. https://doi.org/https:// doi.org/10.1016/j.apradiso.2018.12.020

Dinis, I., Simões, O., Cruz, C. y Teodoro, A. (2019). Understanding the impact of intentions in the adoption of local development practices by rural tourism hosts in Portugal. Journal of Rural Studies, 72, 92-103. https://doi.org/10.1016/j.jrurstud.2019.10.002

Fan, K. H. F., Chang, T. C. y Ng, S. L. (2020). The Batek’s dilemma on indigenous tourism. Annals of Tourism Research, 83, 13. https://doi.org/10.1016/j.annals.2020.102948

Gao, C. y Cheng, L. (2020). Land Use Policy Tourism-driven rural spatial restructuring in the metropolitan fringe: An empirical observation. Land Use Policy, 95(24), 12. https://doi.org/10.1016/j.landusepol.2020.104609

Gobernación de Boyacá. (2018). Dinámicas de ocupación de suelo con relación a los asentamientos humanos. Documento diagnóstico en construcción. https://www.dapboyaca.gov.co/wp-content/uploads/2018/09/DIN\%C3\%81MICAS-DE-OCUPACI\%C3\%93N-DESUELO-CON-RELACI\%C3\%93N-A-LOS-ASENTAMIENTOS-HUMANOS.pdf

Guaita, J. M., Martín, J. M., Salinas, J. A. y Mogorrón-Guerrero, H. (2019). An Analysis of the Stability of Rural Tourism as a Desired Condition for Sustainable Tourism. Journal of Business Research, 100, 165-174.

Haigh, M. (2020). Cultural tourism policy in developing regions: The case of Sarawak, Malaysia. Tourism Management, 81, 11. https://doi.org/10.1016/j.tourman.2020.104166

Hunter, W. C. (2020). Annals of Tourism Research Syncretism and indigenous cultural tourism in Taiwan. Annals of Tourism Research, 82, 13. https://doi.org/10.1016/j.annals.2020.102919 
Landoni, P., Dell'era, C., Frattini, F., Messeni, A., Verganti, R. y Manelli, L. (2020). Business model innovation in cultural and creative industries: Insights from three leading mobile gaming firms. Technovation, 92-93, 11. https://doi.org/10.1016/ j.technovation.2019.102084

Li, J. (2020). Culture and tourism-led peri-urban transformation in China - The case of Shanghai. Cities, 99, 8. https://doi.org/10.1016/ j.cities.2020.102628

Li, J., Bai, Y. y Alatalo, J. (2020). Impacts of rural tourism-driven land use change on ecosystems services provision in Erhai Lake Basin, China. Ecosystem Services, 42, 10. https://doi.org/10.1016/j.ecoser.2020.101081

Liu, S. T. (2020). Comparing the perspectives of municipal tourism departments and cultural departments on urban cultural-tourism development. Journal of Destination Marketing and Management, 16, 8. https://doi.org/10.1016/j.jdmm.2020.100432

Luz, N. (2020). Pilgrimage and religious tourism in Islam. Annals of Tourism Research, 8, 9. https://doi.org/10.1016/j.annals.2020.102915

Marquez, D. L. y Casas, M. (2017). Turismo y desarrollo sostenible. Fundamentación teórica para la construcción de un modelo de desarrollo turístico. Universidad y Sociedad, 9(2), 313-318.

Matiz-León, J. C. (2019). Modelos de temperatura del suelo a partir de sondeos superficiales y sensores remotos para el área geotérmica. Boletín de Geología, 41, 71-88. https://doi.org/10.18273/revbol.v41n2-2019004.RESUMEN

Minnaert, L. (2020). Stakeholder stories: Exploring social tourism networks. Annals of Tourism Research, 83, 102979. https://doi.org/ 10.1016/j.annals.2020.102979

Moreno, V. (2018). Characterization of radon levels in soil and groundwater in the North Maladeta Fault area (Central Pyrenees) and their e ff ects on indoor radon concentration in a thermal spa. Journal of Environmental Radioactivity, 189, 1-13. https://doi.org/ 10.1016/j.jenvrad.2018.03.001

Orgaz, F. y Moral, S. (2016). El turismo como motor potencial para el desarrollo económico de zonas fronterizas en vías de desarrollo. Un estudio de caso. El Periplo Sustentable, (31). http://www.redalyc.org/articulo.oa?id=193449985009

Padilla, J. y Pineda, F. (2019). Análisis de las condiciones para el desarrollo de un programa de ecoturismo comunitario en la ciénaga de Mallorquín barrio La Playa Barranquilla-Atlántico [Trabajo de grado, Universidad de la Costa]. https:// repositorio.cuc.edu.co/handle/11323/3118

Pagliara, F., Aria, M., Russo, L., Corte, V., Della, V. y Nunkoo, R. (2020). Validating a Theoretical Model of Citizens’ Trust in Tourism Development. Socio-Economic Planning Sciences, 35. https://doi.org/10.1016/j.seps.2020.100922

Pavlidis, G. y Markantonatou, S. (2020). Gastronomic tourism in Greece and beyond: a thorough review. International Journal of Gastronomy and Food Science, 47. https://doi.org/10.1016/j.ijgfs.2020.100229

Pilving, T., Kull, T., Suškevics, M. y Viira, A. H. (2019). The tourism partnership life cycle in Estonia: Striving towards sustainable multisectoral rural tourism collaboration. Tourism Management Perspectives, 31, 219-230. https://doi.org/10.1016/j.tmp.2019.05.001

Pyke, J., Pyke, S. y Watuwa, R. (2019). Social tourism and well-being in a fi rst nation community. Annals of Tourism Research, 77, 38-48. https://doi.org/10.1016/j.annals.2019.04.013

Randelli, F. y Martellozzo, F. (2019). Is rural tourism-induced built-up growth a threat for the sustainability of rural areas/ ? The case study of Tuscany. Land Use Policy, 86, 387-398. https://doi.org/10.1016/j.landusepol.2019.05.018

Richards, G. (2018). Management Cultural tourism: A review of recent research and trends. Journal of Hospitality and Tourism Management, 36, 12-21. https://doi.org/10.1016/j.jhtm.2018.03.005

Rodríguez-Gulías, M. J., Fernández-López, S. y Rodeiro-Pazos, D. (2020). Innovation in cultural and creative industries firms with an academic origin (CCI-USOs): The role of regional context. Technovation, 92-93, 102044. https://doi.org/10.1016/ j.technovation.2018.06.007

Rodríguez, D. (2017). Diagnóstico de competitividad del turismo en Boyacá (Colombia). Revista de La Facultad de Ciencias Económica: Investigación y Reflexión, 25(2), 193-205. 
Sam Liu, C. H. y Huang, C. E. (2020). Discovering differences in the relationship among social entrepreneurial orientation, extensions to market orientation and value co-creation - The moderating role of social entrepreneurial self-efficacy. Journal of Hospitality and Tourism Management, 42, 97-106. https://doi.org/10.1016/j.jhtm.2019.12.002

Seyitoglu, F. y Ivanov, S. (2020). A conceptual study of the strategic role of gastronomy in tourism destinations. International Journal of Gastronomy and Food Science, 21, 100230. https://doi.org/10.1016/j.ijgfs.2020.100230

Su, M. M., Wall, G., Wang, Y. y Jin, M. (2019). Livelihood sustainability in a rural tourism destination - Hetu Town, Anhui Province, China. Tourism Management, 71, 272-281. https://doi.org/10.1016/j.tourman.2018.10.019

Terzidou, M. (2020). Re-materialising the religious tourism experience: A post-human perspective. Annals of Tourism Research, 83, 102924. https://doi.org/10.1016/j.annals.2020.102924

Tiberghien, G., Bremner, H. y Milne, S. (2020). Authenticity and disorientation in the tourism experience. Journal of Outdoor Recreation and Tourism, 30, 100283. https://doi.org/10.1016/j.jort.2020.100283

Triviño, J. A. (2020). Evolución del sector turismo y su incidencia en el crecimiento económico del departamento de Boyacá durante el periodo 2000-2016. Fundación Universidad De América, Facultad De Ciencias Económicas y Administrativas. http://52.0.229.99/ bitstream/20.500.11839/7742/1/2161170-2020-1-EC.pdf

Vergori, A. S. y Arima, S. (2020). Cultural and non-cultural tourism: Evidence from Italian experience. Tourism Management, 78, 6. https://doi.org/10.1016/j.tourman.2019.104058

Wang, J., Huang, X., Gong, Z. y Cao, K. (2020). Dynamic assessment of tourism carrying capacity and its impacts on tourism economic growth in urban tourism destinations in China. Journal of Destination Marketing \& Management, 15, 15. https://doi.org/ 10.1016/j.jdmm.2019.100383

Weaver, D., Tang, C. y Zhao, Y. (2020). Annals of Tourism Research Facilitating sustainable tourism by endogenization: China as exemplar. Annals of Tourism Research, 81, 13. https://doi.org/10.1016/j.annals.2020.102890

Wei, C., Dai, S., Xu, H. y Wang, H. (2020). Cultural worldview and cultural experience in natural tourism sites. Journal of Hospitality and Tourism Management, 43, 241-249. https://doi.org/10.1016/j.jhtm.2020.04.011

Wu, Y. C., Lin, S. W. y Wang, Y. H. (2020). Cultural tourism and temples: Content construction and interactivity design. Tourism Management, 76, 8. https://doi.org/10.1016/j.tourman.2019.103972

Nini Johanna Cruz Rodríguez

Universidad Pedagógica y Tecnológica de Colombia (UPTC).

Psicóloga, especialista en Educación y atención a la primera infancia de la Universidad Pedagógica y Tecnológica de Colombia (UPTC). Magister en pedagogía de la Universidad Santo Tomás. Psicóloga de la Fundación Pedagógica Rayuela en Tunja y docente orientadora de la Secretaría de Educación de Boyacá en Motavita (Boyacá).

nijo2323@gmail.com

ORCID: https://orcid.org/0000-0002-6896-5904 